members of the Council had presented their case, expressed himself as in complete sympathy with the object it had in view, and promised to do what he could to get non-pauper patients made eligible for admission to the Metropolitan Asylums Board's ophthalmia institution as had been done for admission to their fever hospitals.

The necessary negotiations and arrangements have been carried out, and now any child attending at a London County Council school, suffering from a contagious eye disease, can be admitted to the ophthalmia school at Swanley, provided accommodation for the class of children for which it was originally intended is not encroached upon.

The Council of British Ophthalmologists may be congratulated upon the success of their intervention in this matter, calculated as it is, not only to restore numerous poor children to health and efficiency, but also to be a benefit to the community by checking the spread of contagious maladies of the eye amongst its members.

\title{
The Preparation of Scientific Papers
}

Those who aspire to write for the medical press have been placed under a debt of gratitude by the Medical Research Council, the publications department of which has recently (January, 1921) issued a brochure entitled "Notes upon the Preparation of Monographs and Reports for Publication by the Council." It embodies much information likely to be useful to such writers, and not easy to come at by the usual channels. The primary object of the pamphlet appears to be to keep the cost of publications and reports down to the lowest possible figure, a precaution rendered the more necessary on account of the rapidly increasing cost of printing. Care should be taken that copy be typewritten, or, when that is not possible, the utmost care should be taken that proper names and titles should be written legibly. In any case, one side only of the paper should be used, and the MS. should be sent packed flat and not rolled or folded. An author should correct his typewritten copy before it leaves his hands. Reference lists, tables, etc., should be sent in on separate sheets of paper, which, so far as may be, should be of uniform size. When quotations are inserted in the text, it is suggested that, if more than four lines long, they should be marked by a vertical line placed on the left-hand side to indicate that they are to be printed in a separate type. New paragraphs should be indicated by indenting the first line of the new paragraph about two inches. Footnotes, as a rule, are not desirable. The best method of keeping and arranging references is by the so-called "Harvard System." (1) According to this plan, all works cited are collected together at the end of an article where they are arranged in alpha- 
betical order according to authors' names, followed by initial and printed in small capitals. (2) This is followed by the date of publication of the work enclosed in brackets. If more than one paper by an author has to be cited for the same year the different papers are distinguished by adding immediately after the date the letters a, b, c, etc. to each. (3) Full title of paper or work itself in Roman type. (4) In writing the title of a journal or other periodical publication the abbreviations employed in the catalogue of the Library in the Surgeon-General's office, United States Army, should be used and such abbreviations be printed in italics. (5) The volume of the journal or other periodical is indicated by an Arabic numeral in clarendon type. (6) The page on which the article begins is indicated by an Arabic numeral. Works by the same author are arranged in chronological order. Obscure or misleading abbreviations should be avoided. A list of abbreviations for units of the metric system is given. If there be any possibility of doubt, "gramme" and "grain" should be spelt in full and no attempt be made to abbreviate them. Where degrees of temperature are referred to, the scale employed should always be specified. To indicate a percentage ' per cent.' should be used and not the symbol $\%$. The familiar caution is repeated, namely, not to place a decimal point at the beginning of a number, e.g., the form .5 should always be replaced by 0.5 . Tables should be prepared on separate sheets, numbered in the order in which they are to be used in the text, and instructions to the printer should be placed in the text to indicate where the tables are eventually to appear. In referring in the text to tables the number should be quoted and such phrases as "the following table" avoided. The number of illustrations should be kept as low as possible. In photographs much essential detail is lost and the inclusion of non-essential detail leads to confusion. Line drawings should be employed in preference to wash drawings or photographs as the cost of line blocks is about half of that of half-tones. Half-tone blocks have also to be printed on special paper which involves further expense. Illustrations should never be inserted in the MS., since they have to be sent to the engravers when the MS. is sent to the printers. They should be numbered consecutively. The descriptions of the figures should be written on separate sheets, but may also be inserted on the illustrations themselves. If drawings are made for reduction, the reference letters may be too small when reduced. It is usually better for the author to insert the lettering lightly in pencil so as to allow the skilled draughtsman employed by the engraver to give it its final form. The notes end by a consideration of the scientific names of animals and plants. 